\title{
PERCEPCIÓN DEL RIESGO DE CÁNCER DE PIEL EN UN HOSPITAL DE ALTURA
}

\author{
RISK PERCEPTION OF SKIN CANCER AT A HIGH-ALTITUDE HOSPITAL \\ Nuria Leticia Huanca-Huirse ${ }^{1,2}$, Joel Sack Roque-Roque ${ }^{1,2}$, Verónica Nélida Laurel-Vargas 1,2, \\ Alan Wenceslao Quispe-Sancho ${ }^{1,2}$
}

\begin{abstract}
RESUMEN
Objetivo: Determinar el nivel de percepción de riesgo de cáncer de piel en una población de Cusco. Métodos: El estudio es observacional de tipo transversal descriptivo y se llevó a cabo en una campaña de despistaje de cáncer de piel en la ciudad de Cusco. El cuestionario aplicado fue diseñado y validado por Morales-Sánchez M y Cols, y mide la percepción de riesgo de cáncer de piel. El valor de alfa de Cronbach reportado es de 0,824. Resultados: La mayoría de pacientes fueron de sexo femenino (64,7\%), de edad promedio fue de 44,9 años (DS: 14,2) y de grado de educación superior (51,0\%). La mediana de horas de exposición solar fue de 4 (IC95\%: 1 - 6), la procedencia más frecuente fue del Cusco $(77,6 \%)$ y con una percepción normal del cáncer de piel $(65,8 \%)$. Conclusión: La percepción de riesgo de cáncer de piel fue normal en la mayoría de la población.
\end{abstract}

Palabras clave: Percepción; Neoplasias cutáneas; Actitudes y práctica en salud (fuente: DeCS BIREME).

\begin{abstract}
Objective: To determine the level of risk perception of skin cancer in a population from Cusco. Methods: It is an observational descriptive cross-sectional study and was aplied in a skin cancer screening campaign in Cusco. The applied questionnaire was designed and validated by Morales-Sánchez M and Cols, and it measures the risk perception of skin cancer. The reported Cronbach's alpha value is 0.824 . Results: The majority of patients were female (64.7\%), average age was 44.9 years (SD: 14.2 ) and the $51.0 \%$ had higher education level. The median hours of sun exposure was $4(95 \% \mathrm{Cl}: 1-6)$, the most frequent origin was from Cusco (77.6\%) and most of the population have normal risk perception of skin cancer (65.8\%). Conclusion: The risk perception of skin cancer was normal in the majority of the population.
\end{abstract}

Key words: Skin neoplasms; Perception; Health behavior; Attitude (source: MeSH NLM).

\section{INTRODUCCIÓN}

La exposición a radiación ultravioleta (RUV) solar prolongada tiene el potencial de dañar las células de la piel y producir neoplasias. La incidencia de cáncer de piel va en incremento a pesar de las estrategias de prevención; es así que la Sociedad Americana de Cáncer estimó 96480 nuevos casos de melanoma invasivo para el $2019^{(1)}$ con una tendencia creciente desde 1999 (2,3). En Perú, el cáncer de piel fue el cuarto tipo de cáncer más frecuente en 2012 y en 2017 fue el segundo ( 7,9\% vs 10,4\%) ${ }^{(4,5)}$. Cada año se diagnostican más de 66000 casos nuevos y se estima que fallecen más de 32000 personas por esta enfermedad ${ }^{(6)}$. La mayoría de tipos de cáncer de piel son altamente curables; sin embargo, el tratamiento no oportuno tiene repercusiones en el paciente como desfiguración y trastornos emocionales ${ }^{(7)}$.

Las medidas preventivas y el diagnóstico temprano de cáncer de piel ayudarían para disminuir los indicadores antes mencionados ${ }^{(8)}$. El cáncer de piel es potencialmente prevenible mediante conductas adecuadas como evitar la exposición solar, utilizar

${ }^{1}$ Escuela Profesional de Medicina Humana, Universidad Nacional de San Antonio Abad del Cusco, Cusco-Perú.

${ }^{2}$ ASOCIEMH CUSCO Asociación Científica de Estudiantes de Medicina Humana del Cusco, Universidad Nacional de San Antonio Abad del Cusco, Cusco-Perú.

Citar como: Nuria Leticia Huanca-Huirse, Joel Sack Roque-Roque, Verónica Nélida Laurel-Vargas, Alan Wenceslao Quispe-Sancho. Percepción del riesgo de cáncer de piel en un hospital de altura. Rev. Fac. Med. Hum. Abril 2020; 20(2):56-60. DOI 10.25176/RFMH.v20i2.2895

Artículo publicado por la Revista de la Facultad de Medicina Humana de la Universidad Ricardo Palma. Es un artículo de acceso abierto, distribuído bajo los términos de la Licencia Creative Commons: Creative Commons Attribution 4.0 International, CC BY 4.0 (https://creativecommons.org/licenses/by/4.0/), que permite el uso no comercial, distribución y reproducción en cualquier medio, siempre que la obra original sea debidamente citada. Para uso comercial, por favor póngase en contacto con revista.medicina@urp.pe 
bloqueador solar, accesorios y ropa protectora ${ }^{(9)}$. El diagnóstico de cáncer de piel es frecuente en personas jóvenes hispanas y la tasa de supervivencia es menor en comparación a la población americana ${ }^{(3,10)}$. Por este motivo, la medición de percepción de cáncer de piel es importante desde edades tempranas e influencia en la adopción de estas medidas para la prevención ${ }^{(11,12)}$. Aunque hay estudios sobre percepción y actitud de prevención de cáncer de piel, el diseño de los cuestionarios empleados tiene algunas deficiencias ${ }^{(13)}$.

La región de los andes de Perú se considera a partir de los 1000 metros sobre el nivel del mar y presenta características climáticas y meteorológicas particulares $^{(14)}$. Una de las características es que los índices de RUV son "extremadamente altos" con un índice de 12 y 18 en estas zonas y de 18 en la ciudad del Cusco ${ }^{(15)}$. La incidencia de melanoma maligno en la ciudad del Cusco es de 13 según el último reporte ${ }^{(16)}$. No se encontraron estudios publicados sobre percepción de cáncer de piel en población altoandina, por ello el objetivo de nuestro estudio fue determinar la percepción de riesgo de cáncer de piel en pacientes que acudieron a una campaña de despistaje de cáncer de piel en un hospital de Cusco.

\section{MÉTODOS}

\section{Diseño}

Estudio observacional de tipo transversal descriptivo.

\section{Población y muestra}

Se aplicó un cuestionario a todos los pacientes asistentes a la campaña por el "Día del Lunar" del hospital de contingencia Antonio Lorena del Cusco ubicado a 3400 msnm. Esta campaña se llevó a cabo el 02 de febrero del 2019 en las instalaciones del mencionado hospital.

La campaña de salud consistió en una atención preventiva a cargo de un especialista en dermatología. Cada atención consistió en un examen dermatológico completo, determinación de fototipo de piel y consejería. La aplicación del cuestionario fue previo al ingreso de los pacientes al consultorio. La información del fototipo de piel fue brindada por el dermatólogo, posterior a cada atención. La metodología de aplicación del cuestionario fue uniformizada en una reunión un día antes de la campaña.

\section{Procedimientos y variables}

Se incluyó a todos los pacientes mayores de edad que aceptaron participar del estudio, previo consentimiento informado. Los pacientes con encuesta incompleta, que respondieron con un patrón irregular o que tuvieran imposibilidad física o mental para responder el cuestionario fueron excluidos del estudio.
El cuestionario aplicado fue diseñado y validado por Morales-Sánchez M y Cols, y mide la percepción de riesgo de cáncer de piel(17). Este cuestionario tiene 18 ítems agrupados en 4 áreas: afectivo (que consta de 5 proposiciones), conductual (que consta de 5 proposiciones), gravedad (que consta de 3 proposiciones), susceptibilidad (que consta de 3 proposiciones) y un indicador de probabilidad (que consta de 2 proposiciones). El valor de alfa de Cronbach reportado es de 0,824(17). De acuerdo al estudio de validación un puntaje normal de percepción de riesgo oscila de 68 a 96 puntos, tener menos puntaje implica una menor percepción de riesgo y tener más de 96 puntos significa una percepción alta de riesgo.

Así mismo, se recogió información adicional como características sociodemográficas: sexo, edad, estado civil, grado de instrucción, ocupación, lugar de procedencia; y características clínicas como horas de exposición solar, fototipo de piel, enfermedad previa de la piel, antecedente familiar de cáncer de piel, uso de accesorios para protección solar e información previa de cáncer de piel.

Cada encuesta fue asignada con un código alfanumérico e ingresada a una base de datos en el programa Microsoft Excel 2013, por tres miembros del equipo de estudio. Un cuarto miembro del equipo realizo control de calidad de la información, revisando la totalidad de los cuestionarios y su correcto llenado en la base de datos.

\section{Análisis estadístico}

El análisis descriptivo se realizó usando frecuencias absolutas y relativas para las variables categóricas. Para describir las variables numéricas se utilizó la media y mediana junto a la desviación estándar (DS) o rango intercuartílico (RIC) respectivamente.

\section{Consideraciones éticas}

La aplicación del instrumento de investigación fue precedida de un consentimiento informado realizado a cada participante, los datos manejados por los investigadores se mantuvieron en la más estricta confidencialidad y no fue publicada información que permita la identificación de ningún participante.

\section{RESULTADOS}

El total de encuestados fue de 56 pacientes. Se excluyeron 4 cuestionarios debido a información incompleta. La mayoría de pacientes fue de sexo femenino $(64,7 \%)$ y de grado de educación superior (51,0\%), la edad promedio fue de 44,9 años (DS: 14,2), la mediana de horas de exposición solar fue de 4 (IC95\%: 1 - 6), la procedencia más frecuente fue del Cusco $(77,6 \%)$ y con una percepción normal del cáncer de piel $(65,8 \%)$ (Tabla 1$)$. 
La mayoría de los participantes tuvo una percepción normal de cáncer de piel $(n=33 ; 63,5 \%)$ seguido de percepción mayor $(n=16 ; 30,8 \%)$. Los participantes con percepción baja fueron tres $(5,8 \%)$.

El fototipos más frecuente fueron los tipos 3 y 4 (46\% cada uno). Así mismo, la mayoría de pacientes con fototipo $3(60,9 \%)$ y fototipo $4(73,9 \%)$ tuvieron una percepción de riesgo de cáncer normal. La mayor proporción de participantes no tuvo alguna enfermedad dermatológica (72,6\%), y tuvo una percepción normal $(59,5)$. Sobre el uso de accesorios para protección solar, el 70,6\% de los participantes usaban algún accesorio, así mismo el 63,9\% de este grupo tuvieron una percepción normal del riesgo de cáncer (Tabla 2).

Tabla 1. Características generales según el puntaje de percepción de riesgo de cáncer de piel.

\begin{tabular}{|c|c|c|c|}
\hline \multirow{3}{*}{ Variables } & \multicolumn{3}{|c|}{ Puntaje de percepción de riesgo de cáncer de piel } \\
\hline & Normal $(n=33)$ & Mayor $(n=16)$ & Menor $(\mathbf{n}=\mathbf{3})$ \\
\hline & $\mathbf{N}(\%)$ & $\mathbf{N}(\%)$ & $\mathbf{N}(\%)$ \\
\hline \multicolumn{4}{|l|}{ Sexo } \\
\hline Masculino & $12(66,7)$ & $6(33,3)$ & 0 \\
\hline Femenino & $20(60,6)$ & $10(30,3)$ & $3(9,1)$ \\
\hline \multicolumn{4}{|l|}{ Estado civil } \\
\hline Soltero & $11(61,1)$ & $7(38,9)$ & $0(0,0)$ \\
\hline Casado & $13(72,2)$ & $4(22,2)$ & $1(5,6)$ \\
\hline Conviviente & $6(85,7)$ & $1(14,3)$ & $0(0,0)$ \\
\hline Viudo & $2(40,0)$ & $3(60,0)$ & $0(0,0)$ \\
\hline \multicolumn{4}{|l|}{ Grado de instrucción } \\
\hline Analfabeto & $1(100,0)$ & $0(0,0)$ & $0(0,0)$ \\
\hline Primaria & $3(42,9)$ & $4(57,1)$ & $0(0,0)$ \\
\hline Secundaria & $11(68,8)$ & $5(31,2)$ & $0(0,0)$ \\
\hline Superior & $17(68,0)$ & $6(24,0)$ & $2(8,0)$ \\
\hline \multicolumn{4}{|l|}{ Ocupación } \\
\hline Ama de casa & $9(56,3)$ & $7(43,7)$ & $0(0,0)$ \\
\hline Estudiante & $4(80,0)$ & $1(20,0)$ & $0(0,0)$ \\
\hline Enfermera & $4(80,0)$ & $0(0,0)$ & $1(20,0)$ \\
\hline Otros & $16(61,5)$ & $8(30,8)$ & $2(7,7)$ \\
\hline \multicolumn{4}{|l|}{ Lugar de procedencia } \\
\hline Cusco ciudad & $25(65,8)$ & $12(31,6)$ & $1(2,6)$ \\
\hline Fuera de Cusco & $7(63,4)$ & $3(27,3)$ & $1(9,1)$ \\
\hline
\end{tabular}


Tabla 2. Características clínicas según el puntaje de percepción de riesgo de cáncer de piel.

\begin{tabular}{|c|c|c|c|}
\hline \multirow{3}{*}{ Variables } & \multicolumn{3}{|c|}{ Puntaje de percepción de riesgo de cáncer de piel } \\
\hline & Normal $(n=33)$ & Mayor $(n=16)$ & Menor $(n=3)$ \\
\hline & $\mathbf{N}(\%)$ & $\mathbf{N}(\%)$ & $\mathbf{N}(\%)$ \\
\hline \multicolumn{4}{|l|}{ Fototipo } \\
\hline Tipo 2 & $2(66,8)$ & $1(33,3)$ & $0(0,0)$ \\
\hline Tipo 3 & $14(60,9)$ & $7(30,4)$ & $2(8,7)$ \\
\hline Tipo 4 & $17(73,9)$ & $6(26,1)$ & $0(0,0)$ \\
\hline Tipo 5 & $0(0,0)$ & $1(100,0)$ & $0(0,0)$ \\
\hline \multicolumn{4}{|c|}{ Enfermedad de la piel } \\
\hline Si & $11(78,6)$ & $2(14,3)$ & $1(7,1)$ \\
\hline No & $22(59,5)$ & $13(35,1)$ & $2(5,4)$ \\
\hline \multicolumn{4}{|c|}{ Uso de accesorio para protección solar } \\
\hline Si & $23(63,9)$ & $10(27,8)$ & $3(8,3)$ \\
\hline No & $10(66,7)$ & $5(33,3)$ & $0(0,0)$ \\
\hline \multicolumn{4}{|c|}{ Información previa de cáncer de piel } \\
\hline Si & $14(63,4)$ & $6(27,3)$ & $2(9,1)$ \\
\hline No & $19(65,5)$ & $9(31,0)$ & $1(3,5)$ \\
\hline
\end{tabular}

La enfermedad dermatológica más frecuente fue la rosácea (33,3\%) seguido de micosis (25,0\%) (Tabla 3).

Tabla 3. Tipos de enfermedad de piel en los encuestados.

\begin{tabular}{lcc} 
& Tipo & $\mathbf{N}(\%)^{*}$ \\
\hline Rosacea & $4(33,3)$ \\
\hline Micosis & $3(25,0)$ \\
\hline Acné & $2(16,7)$ \\
Alergia & $1(8,3)$ \\
Melasma & $1(8,3)$ \\
\hline Melanoma & $1(8,3)$ \\
\hline
\end{tabular}

*Dos pacientes no refirieron el tipo de enfermedad.

\section{DISCUSIÓN}

La percepción fue normal en el $63,5 \%$ y alto en el $30,8 \%$ de los participantes. Esto podría deberse a un adecuado conocimiento del riesgo de cáncer de piel. Se ha demostrado que el nivel de conocimiento influye en la percepción y en el cambio de actitudes en los pacientes $^{(18)}$. El estudio de Alfredo A y cols. reporta que existe poca percepción de riesgo y deficiente conocimiento acerca de los peligros que conlleva la exposición solar irresponsable ${ }^{(19)}$. La mayoría de participantes $(51,0 \%)$ tuvieron nivel superior de educación, por lo que suponemos el acceso información y concientización sobre la enfermedad es mayor.
La mayoría de participantes $(72,6 \%)$ no tuvo enfermedad de la piel, y de este grupo, más del $90 \%$ tuvo una percepción de riesgo normal o alta. Esto podría explicarse por la alta proporción de participantes que usaba medidas de fotoprotección (70,6\%). En un estudio que incluyó veteranos en tratamiento de enfermedad de la piel, reportaron que entendían el riesgo del cáncer de piel pero veían confuso el uso del protector solar y otras medidas ${ }^{(19,20)}$. Así mismo, un estudio realizado en Francia, reporta que el tener un historial cutáneo aumenta el comportamiento fotoprotector ${ }^{(21)}$. La adopción de medidas de fotoprotección depende de la percepción personal de riesgo, la cual en la mayoría de participantes del estudio es normal o alta ${ }^{(22)}$. 
El fototipo predominante en nuestro estudio fue el 3 y 4 (46\% cada uno). Estos fototipos presentan una mayor cantidad de melanina, la cual tiene capacidad de absorber RUV de amplio espectro, antioxidante y removedor de radicales ${ }^{(23)}$. Así mismo, la percepción de cáncer de estos grupos fue normal o alta en más del $90 \%$ de ellos. Este resultado podría explicarse nuevamente por el nivel educativo de los participantes y el acceso a información.

Las limitaciones que presentó el estudio es que incluyó un grupo no representativo, por lo cual los resultados no podrán extrapolarse a otras poblaciones que acudan a otros hospitales de la ciudad. Se sugiere realizar estudios que incluyan a población de los diferentes sistemas de salud. Es el primer estudio realizado en población altoandina que evalúa la percepción de riesgo sobre el cáncer de piel, utilizando una encuesta validada.

\section{CONCLUSIÓN}

La percepción de riesgo de cáncer de piel fue de normal a alta en la mayoría de los pacientes. El nivel de educación podría ser un factor influyente en la percepción de cáncer de piel.

Agradecimiento: Agradecemos a la Dra. M. A. Morales Sánchez por facilitarnos la ficha de recolección de datos que utilizamos en el presente trabajo.

Contribuciones de autoría: Los autores participaron en la génesis de la idea, diseño de proyecto, recolección e interpretación de datos, análisis de resultados y preparación del manuscrito del presente trabajo de investigación.

\section{Financiamiento: Autofinanciado.}

Conflicto de interés: Los autores declaran no tener conflicto de interés en la publicación de este artículo.

Recibido: 05 de marzo 2020

Aprobado: 19 marzo 2020

Correspondencia: Nuria Leticia Huanca Huirse.

Dirección: Av. de La Cultura 773, Cusco 08000 - Perú.

Teléfono: +51957072120

Correo: nuriahuancahuirse@gmail.com

\section{REFERENCIAS BIBLIOGRÁFICAS}

1. American Cancer Society. Cancer Prevention \& Early Detection Facts \& Figures 2019-2020. Atlanta: American Cancer Society; 2019. Disponible en www.cancer.org/research/cancer-facts-statistics/cancer-prevention-earlydetection.html

2. Anderson RN, Kohler BA, Scott S, Lake AJ, Cronin KA, Jemal A, et al. Annual Report to the Nation on the Status of Cancer, part I: National cancer statistics. Cancer. 2018;124(13):2785-800. Disponible en https://www.ncbi.nlm.nih.gov/ pmc/articles/PMC6033186/

3. Perez MI. Skin Cancer in Hispanics in the United States. J Drugs Dermatol. 2019;18(3):117-20. Disponible en https://jddonline.com/articles/ dermatology/S1545961619S0117X

4. Ministerio de Salud DG de E. Análisis de la situación del cáncer en el Perú. Serie Herramientas Metodológicas en Epidemiología y Salud Pública № 015. 2013: 57 Disponible en https://www.dge.gob.pe/portal/docs/asis_cancer.pdf

5. Perú EDEL. Boletín epidemiológico del perú. 2018;2. Disponible en www. dge.gob.pe/portal/docs/vigilancia/boletines/2017/52.pdf

6. Epidemiológica S. Boletín Epidemiológico Del Perú. Minsa [Internet]. 2019;28:1-31. Disponible en: ISSN 2415-076 2\%0Awww.dge.gob.pe

7. Mermelstein RJ, Riesenberg LA. Changing knowledge and attitudes about skin cancer risk factors in adolescents. Health Psychol. 1992;11(6):371-6.

8. de Haas ER, Nijsten T, de Vries E. Population education in preventing skin cancer: from childhood to adulthood. Journal of Drugs in Dermatology: JDD. 2010 Feb:9(2):112-116 Disponible en: europepmc.org/article/med/20214171

9. Kornek T, Augustin M. Prävention von Hauttumoren. JDDG - J Ger Soc Dermatology. 2013;11(4):283-98. Disponible en: onlinelibrary.wiley.com/doi/ abs/10.1111/ddg.12066_suppl

10. Spradlin K, Bass M, Hyman W, Keathley R. Skin cancer: Knowledge, behaviors, and attitudes of college students. South Med J. 2010;103(10):9991003. Disponible en: insights.ovid.com/article/00007611-201010000-00008

11. Defranc A, Van Den Broucke S, Leroy R, Hoppenbrouwers K, Lesaffre $E$, Martens $\mathrm{L}$, et al. Measuring oral health behaviour in Flemish health care workers: An application of the theory of planned behaviour. Community Dent Health. 2008;25(2):107-14. Disponible en:www.researchgate.net/ publication/51411582_Measuring_oral_health_behaviour_in_Flemish health_care_workers_an_application_of_the_Theory_of_Planned_Behaviour

12. Janssen $\mathrm{E}$, Van Osch $\mathrm{L}$, De Vries $\mathrm{H}$, Lechner L. Measuring risk perceptions of skin cancer: Reliability and validity of different operationalizations. $\mathrm{Br} J$ Health Psychol. 2011;16(1):92-112. Disponible en: onlinelibrary.wiley.com/ doi/abs/10.1348/135910710X514120
13. Toro-Huamanchumo CJ, Burgos-Muñoz SJ, Burgos-Muñoz RM, PerezFernandez J, Zentner-Guevara JA, Vargas-Tineo LM, et al. Awareness, behavior and attitudes concerning sun exposure among beachgoers in the northern coast of Peru. PeerJ. 2019;7:e6189. Disponible en: www.ncbi.nlm.nih.gov/ pmc/articles/PMC6338095/

14. Javier Pulgar Vidal . Las ocho regiones naturales del Perú. Terra Brasilis ( Nova Série ). 2003;5(2007):4-5. Disponible en: journals.openedition.org/ terrabrasilis/1027

15. www.senamhi.gob.pe/?\&p=radiacion-uv

16. Gutiérrez Villafuerte CA, Alarcón Isidro EE, Valle R, Calderón G. Epidemiología del melanoma maligno en el Instituto Nacional de Enfermedades Neoplásicas, Perú, 2000-2004. Folia dermatol peru 2007:18(1):2000-4. Disponible en: sisbib.unmsm.edu.pe/BVRevistas/folia/vol18_n1/pdf/a05v18n1.pdf

17. Morales-Sánchez MA, Peralta-Pedrero ML, Domínguez-Gómez MA. Diseño y validación de un cuestionario para medir percepción de riesgo de cáncer de piel. Actas Dermosifiliogr [Internet]. 2014;105(3):276-85. Dispoible en: www.elsevier.es/es-revista-medicina-familia-semergen-40pdf-S0001731013003785

18. Brewer NT, Chapman GB, Gibbons FX, Gerrard M, Mccaul KD, Weinstein ND. Meta-Analysis of the Relationship Between Risk Perception and Health Behavior: The Example of Vaccination. 2007;26(2):136-45. Disponible en psycnet.apa.org/record/2007-03487-002

19. Guerra Castro MM, Alemán Bacallao AD, Román ManriqueY. Fotoprotección y fotodaño en la niñez y la adolescencia. MEDISAN 2018; 22(8): 804-815.15. Disponible en: medisan.sld.cu/index.php/san/article/view/1914/html

20. McGrath JM, Fisher V, Krejci-Manwaring J. Skin Cancer Warnings and the Need for New Preventive Campaigns: A Pilot Study. Am J Prev Med [Internet]. 2016;50(2):e62-3. Disponible en: http://dx.doi.org/10.1016/j. amepre.2015.10.009

21. Stoebner-Delbarre A, Thezenas S, Kuntz C, Nguyen C, Giordanella JP, SanchoGarnier $\mathrm{H}$, Guillot B. Sun exposure and sun protection behavior and attitudes among the French population. Ann Dermatol Venereol[Internet]. 2005;132(89 Pt 1):652-7 Disponible en: doi.org/10.1016/s0151-9638(05)79412-6

22. Ramos C RM. Conocimientos actitudes y practicas eb fotoproteccion y fototipo cutaneo en asistentes a una campaña preventiva del cancer de piel. Callao -Perú. Febrero 2010. Dermatol Peru. 2010;20(1):169-73. Disponible en: sisbib.unmsm.edu.pe/BVRevistas/dermatologia/v20_n3/pdf/a02v20n3.pdf

23. Michaela Brenner VJH. The protective role of melanin against UV. Photochem Photobiol. 2009;84(3):539-49. Disponible en: www.ncbi.nlm.nih gov/pmc/articles/PMC2671032/ 